\title{
Interfaces e usabilidade: organização de critérios para avaliação
}

Interfaces and usability: evaluation criteria

MARIZ, Lorena Karen Praxedes; Bacharel em Design; Universidade Federal do Rio Grande do Norte karen.mariz@gmail.com

BASTOS, Helena Rugai; Doutora; Universidade Federal do Rio Grande do Norte

helenarugai@gmail.com

\section{Resumo}

O artigo apresenta a organização de conjunto de critérios de usabilidade, para avaliação e para o design de interfaces. Apresenta, de maneira resumida, conceitos de Interação HumanoComputador e pesquisa realizada sobre princípios e critérios de usabilidade, a partir da análise de autores da área. O artigo é resultado parcial de uma pesquisa maior, que objetiva estudo dos fundamentos relacionados ao design de interação e à usabilidade. A investigação propõe a avaliação do acesso às informações sobre Unidades de Saúde do Sistema Único de Saúde na cidade do Natal, Rio Grande do Norte, disponíveis no portal da Prefeitura do município. Na primeira etapa do projeto foram estabelecidos critérios de usabilidade, que serviram de base para a análise do portal e para o desenvolvimento de interface. Assim, a partir de avaliação com usuários e da análise de usabilidade do portal foi possível propor projeto, para redesenho das páginas de acesso às informações sobre as Unidades de Saúde do município.

Palavras Chave: Interação Humano-Computador; usabilidade; critérios de usabilidade.

\begin{abstract}
This paper aims to organize usability criteria as a fundamental part in interactive interfaces design projects. It presents partial results of a major research on concepts Human-computer interaction and research on principles and usability criteria, based on analysis of some authors in this field. The major research aims to study theoretical fundamentals on interaction design and usability, with the goal of evaluating the access to Health Units' information of the Sistema Único de Saúde (Unified Health System) in the city of Natal, Rio Grande do Norte, available in Municipal Government Portal. The research method includes the definition of usability criteria, which supported the analysis of the Government Portal. In addition, the research included a survey with users. Thus, based on the results it was proposed the redesign the pages of the site to access the information about the Unified Health System and its Health Units.
\end{abstract}

Keywords: Human-computer interaction; usability; usability criteria. 


\section{Introdução}

A sociedade contemporânea vivencia um rápido desenvolvimento e transformação tecnológica, que geram, cotidianamente, uma maior interação entre as pessoas e as novas tecnologias voltadas para a informação e para a comunicação. Em consequência desse crescimento, a sociedade passa por mudanças culturais, sociais, educacionais, dentre outras, em razão dessas interações e da disseminação de informações que surgem diariamente (PASSOS, 2008; PORTUGAL, 2010). Considerando tal disseminação, Horn (2000) afirma que simplesmente armazenar grandes quantidades de informação em computadores, não resolve nossa necessidade por informação. Para o autor, a questão não tem relação com a disponibilização de um número maior de informações, mas sim a habilidade de apresentar informações corretas, para pessoas certas em momento oportuno e de maneira efetiva e eficiente. Tal preocupação está relacionada à adequação do produto às necessidades do usuário, que envolve a compreensão das informações, a assimilação da interface e do funcionamento do sistema. Hoje, a tecnologia disponível permite a concepção e o desenvolvimento de ambientes com efetiva interação, com respostas rápidas e troca de informações quase instantânea. É nesse contexto que a área de Interação Humano-Computador [IHC] está inserida. Ela privilegia a qualidade de uso desses sistemas interativos e o seu impacto na vida dos usuários, ou seja, a experiência do usuário, a usabilidade, o sistema interativo e o design de interface.

O artigo apresenta organização de um conjunto de critérios de usabilidade, para avaliação e para o design de interfaces. O estudo aqui apresentado é resultado parcial de uma pesquisa maior, que pretendeu analisar conceitos e fundamentos relacionados ao design de interação e à usabilidade. A investigação maior propôs a avaliação do acesso às informações sobre Unidades de Saúde do Sistema Único de Saúde [SUS] na cidade do Natal, RN, disponíveis no portal da Prefeitura do município, com intuito de propor projeto de redesenho das páginas de acesso às informações sobre as Unidades de Saúde. Para atingir tais objetivos, foi necessário o estudo, a partir da revisão bibliográfica, para a compreensão de conceitos e fundamentos, sobre: IHC em David Benyon (2011), Preece, Rogers e Sharp (2013) e Barbosa e Silva (2010); design de interação em Preece, Rogers e Sharp (2013); design digital na obra de Ravier Royo (2008). Para o desenvolvimento de critérios ergonômicos para avaliação de usabilidade, foram utilizados os princípios e regras para o design e para a avaliação de interfaces elaborados por autores como J.M. Christian Bastien e Dominique L. Scapin (1993), Jakob Nielsen (1995), Ben Shneiderman e Catherine Plaisant (2005), Enrico Bertini, Silvia Gabrielli e Stephen Kimant (2006), Santa Rosa e Moraes (2012), Olibário José Machado Neto (2013). Como mencionado, o conjunto de critérios serviram de base tanto para a avaliação das interfaces como para o redenho das páginas de acesso às informações sobre as Unidades de Saúde, no portal da Prefeitura. Outrossim, na etapa de levantamento sobre o objeto estudado, foi conduzida pesquisa de avaliação com usuários do sistema SUS, para levantar as principais questões e dificuldades relacionadas ao acesso às informações.

O método utilizado para o desenvolvimento do projeto seguiu a proposta de Meurer e Szabluk (2010), que tem como base o método de Garrett (2003). O método adaptado de Garret $(2003)^{1}$ apresenta seis etapas de projeto, a saber: Estratégia, Escopo, Estrutura, Esqueleto, Visual

\footnotetext{
${ }^{1}$ No livro The Elements of User Experience: User-centered design for the web (2003) Garret analisa elementos esseciais e aspectos relacionados à experiência do usuário na web. A partir dessa análise, o autor desenvolve um processo, baseado em 5 planos (p. 27 - 34) - Estratégia (Strategy), Escopo (Scope), Estrutura (Structure), Esqueleto (Skeleton), Superfície (Surface), que apresenta como os aspectos e elementos da experiência do usuário podem
} 
e Execução. Justamente na primeira fase de projeto foram estabelecidos critérios de usabilidade, que serviram de base para a análise tanto do site da Prefeitura da cidade do Natal como de outro sistema similar. O conjunto de critérios, de igual maneira, nortearam o desenvolvimento de projeto até a fase de concepção de modelos de baixa fidelidade.

\section{Interação Humano-Computador}

Não obstante a qualidade das informações e as oportunidades de acesso, é importante especial atenção ao desenvolvimento de produtos interativos, sobretudo no ambiente virtual. Tal preocupação está relacionada à adequação do produto às necessidades do usuário, que envolve a assimilação da interface e do funcionamento do sistema.

O desenvolvimento de aplicativos para o ambiente virtual pressupõe especial atenção à adequação das interfaces às necessidades dos usuários, quanto à compreensão, assimilação e disposição das informações e das funcionalidades do sistema, o que inclui acesso às funções e aos dados. A partir da assimilação, o usuário executa ações específicas, para ter acesso às informações apresentadas na interface, com o propósito de obter respostas do sistema. Em outras palavras, as interfaces devem permitir que os usuários acessem informações desejadas e funcionalidades do sistema, em qualquer dispositivo como computador, smartphones, tablets, estejam eles conectados ou não a uma rede. De maneira mais abrangente, os sistemas interativos, desde a concepção ao desenvolvimento, devem permitir que os usuários alcancem os objetivos almejados (BARBOSA; SILVA, 2010).

Os estudos e consequente desenvolvimento de tecnologias apropriadas para a efetiva interação, permitem rápido acesso às informações, bem como respostas imediatas dos sistemas. Como mencionado, é nesse contexto que a área de IHC está inserida. Ela privilegia a qualidade de uso desses sistemas interativos e o seu impacto na vida dos usuários (BARBOSA; SILVA, 2010). Outrossim, a IHC privilegia a experiência do usuário, a usabilidade, o sistema interativo e o design de interface. No campo do IHC o design de interface, meio pelo qual o usuário se comunica com o sistema interativo, envolve a apresentação da informação, interação, uso, experiência, entre outros. É possível perceber que o design, no contexto da interação, pode contribuir significativamente para a melhoria das interfaces, promovendo o uso e a compreensão da interface.

A IHC é um campo de estudos interessado no projeto, na implementação e na avaliação de sistemas computacionais interativos para uso humano, além dos fenômenos relacionados a esse uso (BARBOSA; SILVA, 2010). Estabeleceu-se como um campo de estudo importante no início da década de 1980 (BENYON, 2011). Segundo o autor, no início da década de 1990, surgiu a World Wide Web [web] estabelecendo o design de sites como uma nova área. Também a arquitetura e o design da informação surgiram como áreas importantes de estudo e as questões sobre usabilidade se tornaram importantes no mundo da web. Designers de interação se tornaram necessários, assim como os engenheiros de software, para proporcionar às pessoas experiências interessantes entre eles e os sistemas. A construção e o uso de um artefato, apesar de serem aspectos

orientar o planejamento, o controle e o desenvolvimento de projetos centrados no usuário. O artigo de Meurer e Szabluk (2010), por sua vez, apresenta uma adaptação do processo de Garret (2003), em forma de método com 6 macro etapas, que incluem subfases, indicando técnicas, instrumentos e ferramentas para desenvolvimento de projetos de design. Meurer e Szabluk incluem no processo a etapa de Execução e recomendam a confecção de "modelo funcional navegável" nas palavras dos autores (2010, p.7). 
articulados, ocorrem em contextos diferentes e apresentam lógicas distintas. Nesse contexto, um sistema interativo considerado com alta qualidade de construção, nem sempre apresenta uma boa avaliação de usabilidade ou vice-versa.

A computação tem como prioridade a qualidade da construção, ou seja, conceber primeiro representações de dados, arquitetura do sistema e tudo aquilo que permite o funcionamento do sistema interativo. Pouca atenção é dedicada ao que é externo ao sistema. Trata-se de uma abordagem de "dentro para fora" (Figura 1, ilustração A). Neste caminho, os usuários têm que se adaptar ao sistema, porém o mundo externo ao sistema nem sempre se adapta de forma fácil e rápida.

Figura 1 - Abordagem "dentro para fora" (A) e "fora para dentro" (B)

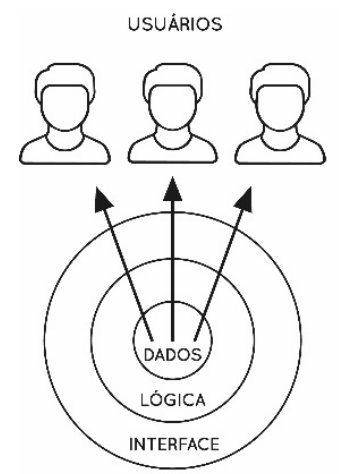

A

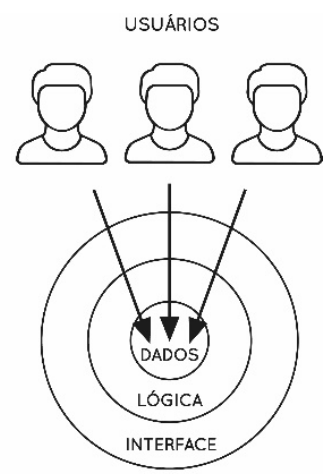

B

Fonte: adaptado de Barbosa e Silva (2010, p. 9).

A área de IHC busca seguir uma abordagem "de fora para dentro" (Figura 1, ilustração B), centrada nos atores envolvidos. Nesta perspectiva, para o desenvolvimento de projeto é necessário iniciar estudo sobre os objetivos, as atividades, as motivações do usuário, o que inclui o contexto de uso, para posterior identificação de oportunidades de intervenção na interface ou no sistema (BARBOSA; SILVA, 2010). Em outras palavras, as abordagens IHC focam no uso e no contexto de uso de sistemas interativos, envolvendo os usuários no processo, e com o design de sistemas interativos, que se preocupa com a criação de experiências interativas, acessíveis e adequadas ao uso (BENYON, 2011).

Um fator que influencia a interação e, consequentemente, o uso, são as características humanas, físicas e cognitivas. A interação com um sistema computacional interativo que lida com informações, requer capacidade cognitiva para processar as informações e aprender a utilizá-las. Influencia, outrossim, a maneira como as pessoas se comunicam e interagem entre si ou com sistemas. Isto porque estas formas de interação são, habitualmente, reproduzidas quando estas pessoas utilizam um sistema computacional interativo (REEVES; NASS, 2003, apud BARBOSA; SILVA, 2010). Desse modo, é natural, em projetos de interação, a utilização de modelos conceituais já conhecidos pelos usuários, para facilitar o aprendizado e a compreensão.

\subsection{Usabilidade}

Por meio da interface, o usuário pode se comunicar com artefatos (físicos ou digitais). Se um design não é funcional e não facilita a utilização do artefato, esta função fica comprometida. 
Assim, "a usabilidade ou capacidade e facilidade de uso de um aparelho, de uma instalação, de um formulário ou de um site é uma característica implícita no campo do design" (ROYO, 2008, p.90). O autor explicita que a usabilidade depende de três fatores:

- Do usuário que reconhecerá, entenderá e manipulará o aparelho em função de seus conhecimentos e da capacidade de uso que o aparelho oferece;

- Do aparelho, que será entendido e manipulado pelo usuário de acordo com o design da interface que o designer proporciona (sob o ponto de vista funcional e visual);

- Do contexto que influirá e em alguns casos definirá o uso do aparelho, modificando o seu significado. (2008, p.94)

Nielsen (1993, p.23-37) aborda a usabilidade de acordo com um conjunto de fatores, que auxiliam a qualificar quão bem uma pessoa interage com um produto interativo. Os fatores de usabilidade são, de acordo com o autor: facilidade de aprendizado (learnability) e de recordação (memorability), eficiência (efficiency of use), segurança no uso (safety) e satisfação do usuário (satisfaction).

Preece, Rogers e Sharp (2013) também abordam esses critérios como metas de usabilidade e adicionam a eficácia e a utilidade. Para Barbosa e Silva (2010) a facilidade de aprendizado tem relação ao tempo e ao esforço necessários, para que o usuário aprenda a usar o sistema. As pessoas esperam que o sistema interativo oferecido seja simples, fácil e rápido de aprender.

A facilidade de recordação envolve o esforço cognitivo necessário para lembrar como o sistema funciona, depois de tê-lo utilizado. Essa capacidade de memorização permite que o usuário se recorde de maneira quase imediata, o que evita erros ao utilizar o sistema. Já a eficiência de um sistema interativo, tem relação ao tempo necessário para finalizar uma tarefa.

Quanto à segurança no uso, Barbosa e Silva (2010) argumentam que é necessário evitar situações indesejadas e, caso aconteça algum problema, o próprio sistema deve conter funções, que auxiliem o usuário a solucionar ou a superar tais situações. Outro aspecto importante, que também tem relação com os demais, é a satisfação do usuário que está associada ao efeito do uso do sistema sobre as emoções do usuário. Diante dos diferentes contextos, locais e situações de uso, a maneira como o usuário utiliza o sistema interativo pode ter efeito emocional. Tal aspecto está relacionado a um fator distinto, o da experiência do usuário. Todos esses aspectos devem ser considerados em processos de avaliação de interfaces interartivas, assim como para o desenvolvimento de projeto.

\section{Organização de critérios de usabilidade para avaliação e desenvolvimento de interfaces interativas}

A avaliação de usabilidade da interface é um processo que busca analisar a interface, visa garantir se o sistema funciona adequadamente e se atende às expectativas dos usuários. Assim, nessas avaliações o objetivo maior é avaliar o acesso às funcionalidades disponibilizadas, além de identificar problemas de design (MACHADO NETO, 2013).

Como mencionado, vários autores desenvolveram princípios para o design de interfaces de sistemas interativos para nortear as avaliações. Para este trabalho apresentamos os princípios dos autores Bastien e Scapin (1993), Jakob Nielsen (1995), Shneiderman e Plaisant (2005) e Bertini et al. (2006), com o intuito de fazer uma comparação entre as diretrizes de usabilidade apresentadas por cada um deles. Tal comparação serviu de base para a organização dos critérios, formando uma 
proposta de critérios para a avaliação de interfaces. O diagrama 1 apresenta as diretrizes dos autores mencionados.

Diagrama 1 - Síntese dos princípios, heurísticas, regras e critérios de Bastien e Scapin (1993), Nielsen (1995), Shneiderman e Plaisant (2005), Bertini et al. (2006).

\begin{tabular}{l}
\hline BASTIEN e SCAPIN (1993) \\
\hline Condução \\
\hline Presteza \\
\hline $\begin{array}{l}\text { Agrupamento/distinção de itens pela } \\
\text { localização }\end{array}$ \\
\hline Agrupamento/distinção pelo formato \\
\hline Feedback imediato \\
\hline Legibilidade \\
\hline Carga de trabalho \\
\hline Concisão \\
\hline Açães mínimas \\
\hline Densidade informacional \\
\hline Controle explícito \\
\hline Ação explíita do usuário \\
\hline Controle pelo usuário \\
\hline Adaptabilidade \\
\hline Flexibilidade \\
\hline Consideração da experiência do usuário \\
\hline Gestão de erros \\
\hline Proteção contra os erros \\
\hline Qualidade das mensagens de erro \\
\hline Correção de erros \\
\hline Homogeneidade / Consistência \\
\hline Significados de códigos \\
\hline Compatibilidade \\
\hline
\end{tabular}

\begin{tabular}{|c|c|}
\hline JAKOB NIELSEN (1995) & SHNEIDERMAN (2005) \\
\hline Visibilidade do status do & Esforce-se pela consistência \\
\hline Equivalência entre o sistema e & $\begin{array}{l}\text { Permita que usuários experien- } \\
\text { tes possam usar atalhos }\end{array}$ \\
\hline & Ofereça feedback informativo \\
\hline Controle do usuário e & \multirow[b]{2}{*}{$\begin{array}{l}\text { Projete diálogos que indiquem } \\
\text { término da ação }\end{array}$} \\
\hline liberdade & \\
\hline Consistência e padrões & \\
\hline Prevenção de erros & $\begin{array}{l}\text { Previna erros e possibilite } \\
\text { correçōes simples }\end{array}$ \\
\hline $\begin{array}{l}\text { Reconhecimento ao invés de } \\
\text { memorização }\end{array}$ & $\begin{array}{l}\text { Permita que ações sejam } \\
\text { desfeitas facilmente }\end{array}$ \\
\hline Flexibilidade e eficiência de uso & $\begin{array}{l}\text { Ofereça um local interno de } \\
\text { controle }\end{array}$ \\
\hline Estética e design minimalista & \multirow{4}{*}{$\begin{array}{l}\text { Reduza a sobrecarga da memó- } \\
\text { ria de curta duração }\end{array}$} \\
\hline Ajudar os usuários a reconhecer, & \\
\hline $\begin{array}{l}\text { diagnosticar e recuperar ações } \\
\text { erradas }\end{array}$ & \\
\hline Ajuda e documentação & \\
\hline
\end{tabular}

BERTINI ET AL. (2006)

Visibilidade do status do sistema e facilidade de encontrar o dispositivo móvel

Compatibilidade entre o sistema e o mundo real

Consistência e mapeamento

Boa ergonomia e design minimalista

Facilidade de entrada de dados, legibilidade e capacidade de assimilação

Flexibilidade, eficiência de uso e personalização

Flexibilidade, eficiência de uso e personalização

Convenções estéticas, sociais e de privacidade

Gerenciamento de erros realístico

Fonte: autores.

Bastien e Scapin (1993) apresentam oito critérios de usabilidade subdivididos em dezoito subcritérios, que incorporam considerações ergonômicas ao processo de avaliação da usabilidade da interface. Tal sistema visa reduzir a redundância na identificação e na classificação das qualidades e problemas ergonômicos da interface interativa (CYBIS, BETIOL, FAUST, 2010; SANTA ROSA, MORAES, 2012).

Jakob Nielsen (1995), por sua vez, expõe dez heurísticas para nortear e para evitar erros nas interfaces. São princípios desenvolvidos sem um contexto específico de uso, mas de uma forma mais ampla, guiam as análises das interfaces de sistemas interativos variados. Já Shneiderman e Plaisant (2005) propõe oito "regras de ouro" para o design de interfaces, que podem ser aplicadas na maioria dos sistemas interativos. Estas são regras para o desenvolvimento de produtos centrados no usuário (SANTA ROSA; MORAES, 2012).

Bertini et al. (2006) elaboraram um conjunto de princípios tendo como base as heurísticas de Nielsen, adaptadas para o contexto das interfaces dos dispositivos móveis, por conta da interação nesses dispositivos ser diferenciada (MACHADO NETO, 2013). Os princípios de Bertini et al. (2006) são elaborados de acordo com os critérios, as heurísticas e regras abordados por Bastien e Scapin (1993), por Nielsen (1993; 1995) e por Shneiderman e Plaisant (2005). Eles foram 
adaptados à realidade dos dispositivos móveis, integrando aspectos importantes para a usabilidade em telas menores, o que corrobora a adição do subcritério de legibilidade, abordado por Bastien e Scapin (1993), e o de flexibilidade, que também é abordado por Jakob Nielsen (1995) juntamente com a eficiência de uso (BERTINI et al., 2006).

Pode-se observar que outras diretrizes estão presentes em quase todas as listas dos autores, como a questão do feedback, a necessidade de minimizar erros, consistência, estética, flexibilidade aos diferentes usuários, redução da sobrecarga cognitiva, entre outras. O diagrama 2 apresenta os princípios e os diretrizes semelhantes, identificados por cores.

Diagrama 2 - Identificação dos princípios similares entre autores.

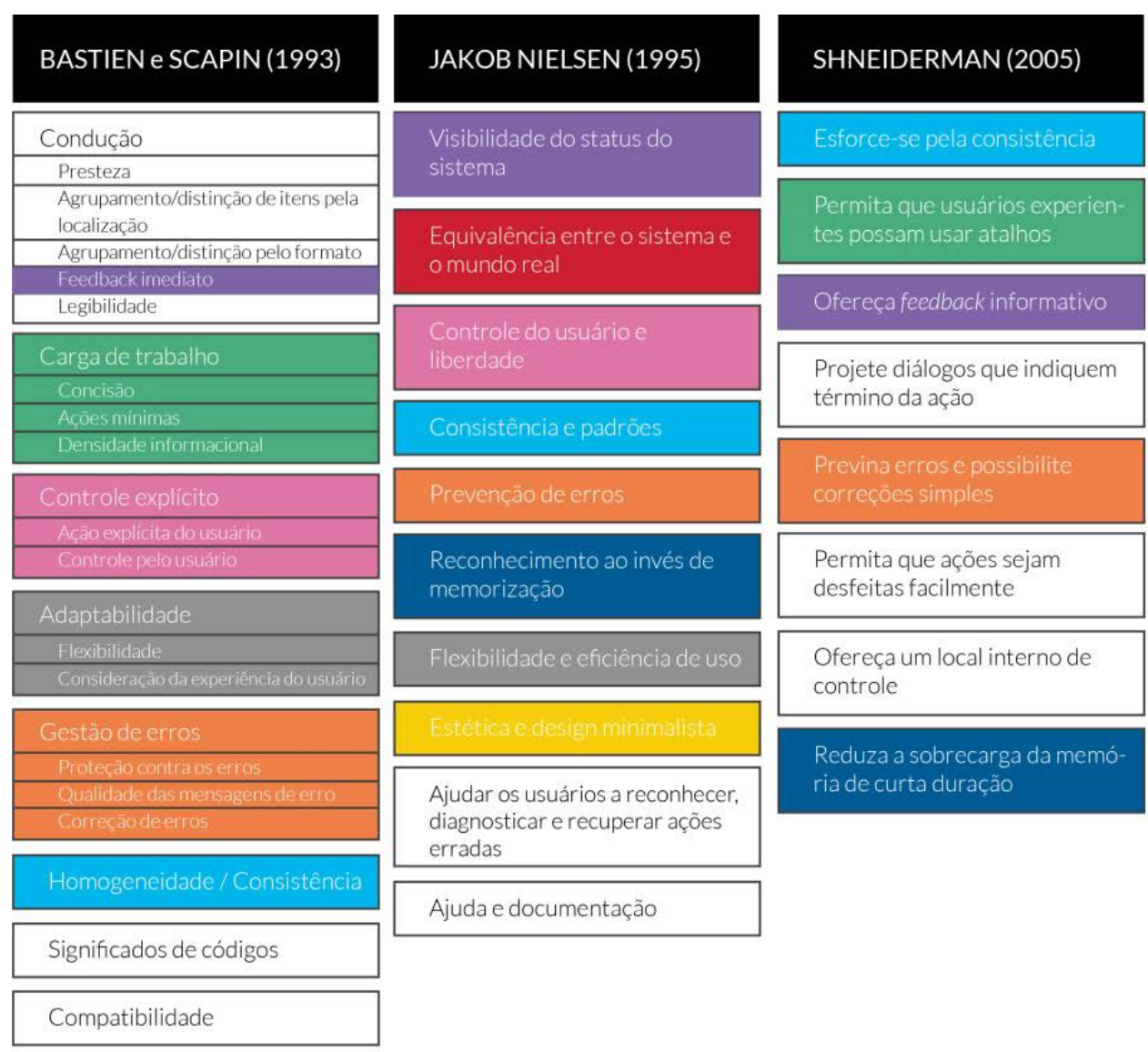

\section{BERTINI ET AL. (2006)}

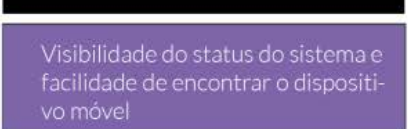

Compatibilidade entre o sistema e o mundo real
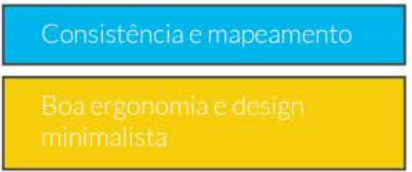

Facilidade de entrada de dados, legibilidade e capacidade de assimilação

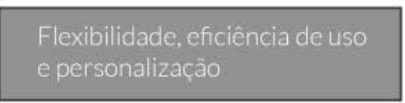

Flexibilidade, eficiência de uso e personalização

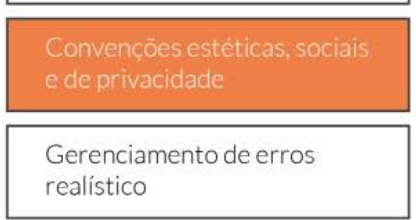

Legenda - Princípios semelhantes apresentadas pelos autores

\begin{tabular}{ll}
$\begin{array}{l}\text { Visibilidade do status do } \\
\text { sistema }\end{array}$ & $\begin{array}{l}\text { Princípio encontrado nos quatro autores: Bastien e Scapin (1993); Nielsen } \\
\text { (1995); Shneiderman e Plaisant (2005); Bertini et al. (2006). }\end{array}$ \\
\hline Gestão de erros & $\begin{array}{l}\text { Princípio encontrado nos quatro autores: Bastien e Scapin (1993); Nielsen } \\
\text { (1995); Shneiderman e Plaisant (2005); Bertini et al. (2006). }\end{array}$ \\
\hline Consistência e padrões & $\begin{array}{l}\text { Princípio encontrado nos quatro autores: Bastien e Scapin (1993); Nielsen } \\
\text { (1995); Shneiderman e Plaisant (2005); Bertini et al. (2006). }\end{array}$ \\
\hline Adaptabilidade & $\begin{array}{l}\text { Princípio encontrado em três autores: Bastien e Scapin (1993); Nielsen (1995); } \\
\text { Bertini et al. (2006). }\end{array}$ \\
\hline Carga de trabalho & $\begin{array}{l}\text { Princípio encontrado em dois autores: Bastien e Scapin (1993); Shneiderman e } \\
\text { Plaisant (2005). }\end{array}$ \\
\hline $\begin{array}{l}\text { Controle do usuário e } \\
\text { liberdade }\end{array}$ & Princípio encontrado em dois autores: Bastien e Scapin (1993); Nielsen (1995). \\
\hline
\end{tabular}




\section{Ergonomia e design \\ Redução da sobrecarga da memória de curta duração Compatibilidade}

Princípio encontrado em dois autores: Nielsen (1995); Bertini et al. (2006).

Princípio encontrado em dois autores: Nielsen (1995); Bertini et al. (2006).

Princípio encontrado em dois autores: Nielsen (1995); Bertini et al. (2006).

Fonte: autores.

A partir da comparação e da análise desses princípios, heurísticas, regras e critérios discutidos pelos autores, observamos as diretrizes comuns entre eles, todas elas relevantes para a avaliação de interfaces de sistemas interativos. Consideramos, para consubstanciar a elaboração de lista única com as principais diretrizes, o contexto de operação dos sistemas e os critérios ergonômicos a priorizar em uma avaliação de usabilidade, indicado por Cybis, Betiol e Faust (2010, p. 49 e 50), a saber:

- compatibilidade;

- densidade informacional;

- legibilidade;

- experiência do usuário.

Tendo como base o estudo de Cybis et al. (2010), a experiência do usuário é um critério relevante, pois um sistema interativo pode atender usuários experientes e novatos no processo de utilização da interface. Níveis diferentes de interação, também apresentam diferentes necessidades de informação (SANTA ROSA; MORAES, 2012). Dentre os autores analisados, Bastien e Scapin (1993), abordam esse ponto no critério da adaptabilidade. Além de tais aspectos, há a questão da acessibilidade para um conjunto amplo de usuários. Para Barbosa e Silva (2010), acessibilidade está relacionada com a capacidade de o usuário acessar o sistema e interagir com ele sem que a interface imponha obstáculos. Preece, Rogers e Sharp (2013) mencionam, que as orientações fornecidas pela legislação, com relação à acessibilidade às pessoas com deficiência, podem ser utilizadas como diretrizes para verificar a interface do sistema. Portanto, a direção fornecida pela legislação deve ser um princípio para análise da interface, visto que se deve permitir o acesso à informação, também por meios digitais, para pessoas com restrições.

Com base no levantamento e nos estudos mencionados, foram compilados os dados e as informações e, resultante dessa análise, foi elaborada uma lista, que apresenta um conjunto de critérios para a avaliação de usabilidade e para o desenvolvimento de interfaces interativas (Tabela 1), adaptados de Bastien e Scapin (1993), Nielsen (1995), Shneiderman e Plaisant (2005), Bertini et al. (2006).

O processo de organização da listagem de critérios, requereu a especificação de subcritérios e a descrição destes princípios, indicados na Tabela 1, com intuito de facilitar sua aplicação em avaliações de usabilidade e para o desenvolvimento de projeto. Os 10 critérios utilizados são:

- Condução, que inclui os princípios de presteza, agrupamento de itens pela localização e pelo formato, feedback imediato, legibilidade, ajuda e documentação.

- Visibilidade do status do sistema.

- Consistência e padrões. 
- Gestão de erros, que inclui: proteção contra erros, qualidade das mensagens de erro e correção de erros.

- Adaptabilidade que propõem a avaliação de flexibilidade e leva em conta a experiência do usuário.

- Ergonomia e design.

- Redução da sobrecarga de memória de curta duração.

- Carga de trabalho que inclui aspectos como: concisão, ações mínimas para acesso às informações e densidade informacional.

- Compatibilidade.

- Acessibilidade.

Tabela 1 - Critérios ergonômicos para a avaliação de usabilidade

\begin{tabular}{ll}
\hline Critérios Ergonômicos & Descrição \\
\hline 1 Condução & $\begin{array}{l}\text { Tem relação com os meios disponíveis para aconselhar, orientar, } \\
\text { informar e conduzir o usuário no processo de interação com o } \\
\text { computador. }\end{array}$ \\
\hline 1.1 Presteza & $\begin{array}{l}\text { Refere-se às informações que permitem ao usuário identificar o } \\
\text { estado ou o contexto durante a interação. Um sistema prestativo guia } \\
\text { e orienta o usuário, livra-o de aprender uma série de comandos e } \\
\text { diminui a ocorrência de erros. }\end{array}$ \\
\hline
\end{tabular}

1.2 Agrupamento ou distinção de itens pela localização

1.3 Agrupamento ou distinção de itens pelo formato

\subsection{Feedback imediato}

1.5 Legibilidade

1.6 Ajuda e documentação

2 Visibilidade do status do sistema

3 Consistência de padrões

4.1 Proteção contra erros
Tem relação com o posicionamento relativo dos elementos e itens na interface.

Refere-se às características gráficas (formato, cor, códigos) que contribuem para a identificação e o reconhecimento das relações entre os elementos ou classes de elementos.

A qualidade e a rapidez de feedback são dois fatores-chave para estabelecer a confiança e a satisfação do usuário, bem como para a compreensão das informações emitidas pelo sistema.

Tem relação com a qualidade da disposição, organização e características de configuração dos elementos que compõem a interface. O desempenho aumenta quando a apresentação da informação na interface leva em conta aspectos cognitivos e perceptivos dos usuários

Diz respeito às informações que contribuem para compreender as funções do sistema. O sistema deve prover ajuda e documentação e as informações relacionadas devem ser fáceis de encontrar, focadas nas atividades do usuário, organizadas em tarefas concretas e objetivas.

O sistema deve manter os usuários informados sobre todo o processo de interação e de acessos.

Tem relação com os padrões estabelecidos na interface, o que inclui a o uso da linguagem verbal, linguagem visual ou sonora. Leva-se em consideração as convenções habitualmente adotadas nos sistemas. Deve-se evitar, igualmente, uso de diferentes termos, ícones ou imagens que indicam a mesma função.

Refere-se a todos os mecanismos que permitem evitar ou reduzir a ocorrência de erros e, caso ocorram devem conduzir à correção imediata.

Diz respeito aos meios disponíveis ao usuário que têm o objetivo de permitir a correção dos erros cometidos no processo de acesso às funções ou informações do sistema. 
4.2 Qualidade das mensagens de erro

4.3 Correção dos erros

5 Adaptabilidade

5.1 Flexibilidade

5.2 Experiência do usuário

6 Ergonomia e design

7 Redução da sobrecarga da memória de curta duração

8 Carga de trabalho

8.1 Concisão

8.2 Ações mínimas

8.3 Densidade informacional

9 Compatibilidade

10 Acessibilidade
Tem relação com a pertinência, à legibilidade e à exatidão das informações apresentadas ao usuário, a respeito da natureza do erro cometido e sobre ações para corrigi-lo.

Diz respeito aos meios disponíveis ao usuário, que permitam a correção do erro.

A capacidade de o sistema reagir de acordo com o contexto, com as necessidades e as preferências do usuário.

Tem relação com os meios que o usuário dispõe para personalizar a interface em função de suas estratégias de trabalho e/ou seus hábitos, além das exigências das tarefas a serem realizadas.

Diz respeito aos meios disponíveis que levam em conta a experiência do usuário.

A comunicação entre usuário e sistema devem privilegiar informações relevantes e funções necessárias. Toda a unidade de informação extra em um diálogo compete com unidades relevantes, o que prejudica a visibilidade relativa das funções importantes.

O sistema deve prever interface de fácil memorização. Para tanto, requer uma estrutura e organização para relacionar elementos e para facilitar a memorização das informações e das funções da página.

Diz respeito à complexidade das tarefas e atividades requeridas para interação no sistema. Deve-se considerar tarefas fáceis e objetivas

Tem relação a capacidade da memorização de curta duração. Funções e tarefas objetivas são concisas.

Refere-se à quantidade de ações necessárias para a realização de uma tarefa ou atividade no sistema, para evitar sobrecarga de trabalho e erros durante o acesso às informações e funcionalidades do sistema. Tem relação com a carga de trabalho aplicada para a identificação e compreensão do conteúdo informacional, sob o ponto de vista perceptivo e cognitivo.

Diz respeito a relação harmônica entre as características do usuário memória, à percepção, os hábitos, habilidades, idade, entre outras - e os atributos das tarefas, o que também inclui a organização de inputs/ outputs.

Leva-se em conta entraves ou obstáculos que dificultam ou impossibilitam o acesso, a recepção e a transmissão das mensagens e informações, considerando necessidades específicas de usuários.

Fonte: autores.

Como mencionado, tais critérios foram utilizados para avaliação do acesso às informações sobre Unidades de Saúde do Sistema Único de Saúde na cidade do Natal, RN, disponíveis no portal da Prefeitura do município. A partir de avaliação com usuários e da análise de usabilidade do portal foi possível propor projeto, para redesenho das páginas de acesso às informações sobre as Unidades de Saúde do município.

\section{Desenvolvimento do projeto}

Apesar do artigo privilegiar a apresentação do processo realizado para a organização dos critérios para a avaliação de usabilidade do sistema estudado, apresentamos aspectos e métodos que conduziram o processo de desenvolvimento de projeto.

O sistema de saúde público (Sistema Único de Saúde), apresenta um ambiente online (a página da Secretaria da Saúde no site da Prefeitura de Natal, RN) com o intuito de auxiliar os 
usuários disponibilizando informações diversas relacionadas ao sistema de saúde do município. Porém, algumas informações não suprem a necessidades mais específicas dos usuários, como a falta de informação sobre localidade de algumas Unidades de saúde, e as informações presentes, não são apresentadas de maneira clara. Outro aspecto relevante levantado é a difícil localização das Unidades de Saúde. Nesse âmbito, foi feito um questionário para levantar dados, com usuários do Sistema Único de Saúde do município. A maioria dos entrevistados conhecem as Unidade de Saúde na região onde mora, porém, os usuários desconhecem a localização das demais Unidades nas outras regiões da cidade. Para ter acesso a essas informações, os usuários buscam na Internet, por telefone e com outras pessoas, como, vizinhos, amigos e servidores das Unidades. Ademais, na entrevista realizada, os entrevistados admitiram preferir a busca em site ou dispositivos móveis, mas que o atual sistema não disponibiliza de maneira clara tais informações. Para além da entrevista realizada, os pesquisadores realizaram a análise do sistema, buscando o percurso necessário para acessar informações sobre o Sistema Único de Saúde, o que incluiu a verificação sobre a Unidades e os serviços prestados por cada uma delas. A avaliação revelou tanto a dificuldade de acesso como a falta de informação sobre as Unidades do município, desrespeitando os critérios de usabilidade estabelecidos (Quadro 1).

A partir do levantamento mencionado e com base nos critérios estabelecidos foi desenvolvido projeto de aplicativo, que, num primeiro momento pode ser acessado pelo portal da Prefeitura, para posteriormente adaptação de aplicativo destinado a dispositivos móveis.

O método utilizado como guia para o desenvolvimento do projeto foi o proposto por Meurer e Szabluk (2010), que é voltado para projetos de design de interação. Sua estrutura tem como base o método de Garrett (2003) e apresenta seis etapas projetuais: Estratégia, Escopo, Estrutura, Esqueleto, Visual e Execução (Diagrama 3).

Diagrama 3 - Esquema das etapas do projeto.

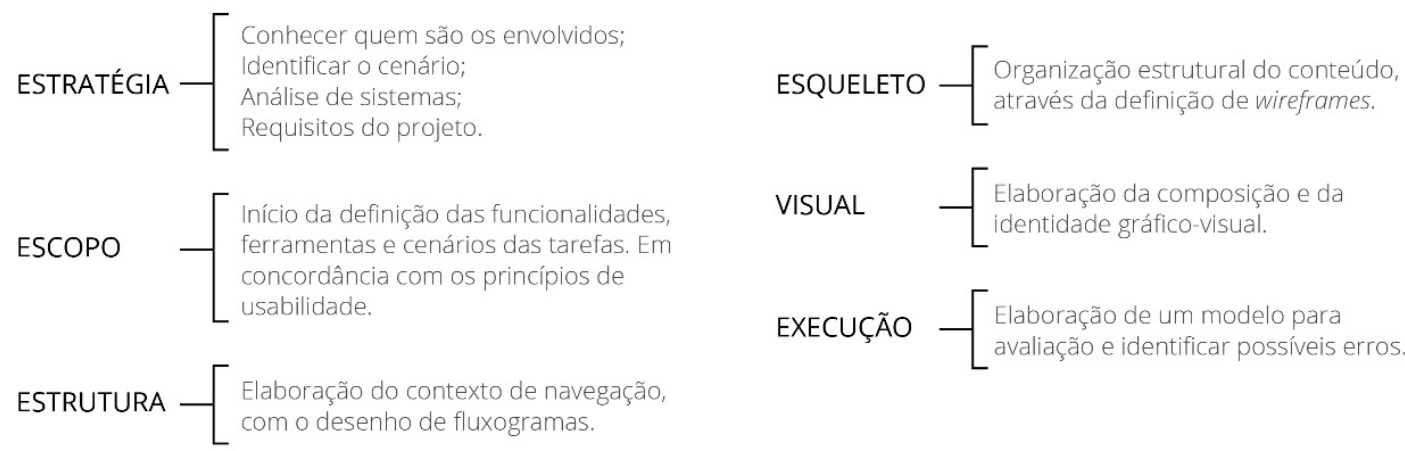

Fonte: adaptado do método de Garrett (2003) apresentado por Santa Rosa e Moraes (2012).

A etapa de Estratégia consiste na identificação do contexto do projeto. Para isso, foram analisados o cenário e o perfil dos usuários, com base nas informações obtidas a partir das entrevistas realizadas com usuários e da análise do processo de avaliação analítica descritas. Tal análise permitiu a definição de requisitos e restrições de projeto, sempre respeitando os critérios estabelecidos e apresentados no Quadro 1 e os objetivos propostos para o desenvolvimento do site. Seguem os requisitos gerais levantados:

- Haver um link de redirecionamento do site da Prefeitura para o site de busca pelas 
Unidades de Saúde, o Natal Saúde;

- Ao final da pesquisa, saber o nome e o endereço da Unidade de saúde, serviços ofertados e o caminho para chegar lá;

- Incorporar mapa do Google maps;

- Associar os elementos visuais à fonte utilizada, para que sejam compreendidos;

- Website sem rolagem horizontal;

- Utilizar linguagem clara para os usuários;

- Estabelecer percurso intuitivo para se chegar à informação desejada (com poucos clicks);

- Possibilitar o aumento da fonte e o alto contraste;

- Fornecer os serviços de cada Unidade de Saúde (UBS, USF, Unidades Especializadas);

Na etapa denominada Escopo, foi iniciada a definição das funcionalidades, ferramentas e cenários das tarefas. É a primeira etapa em que foram geradas alternativas. Assim, as soluções foram elaboradas em concordância com os critérios e subcritérios de análise, para evitar os problemas de usabilidade que possam comprometer a realização das tarefas.

Seguindo o método de projeto, na fase Estrutura foi elaborado o contexto navegacional do site. Nesta etapa foram geradas alternativas de fluxogramas das tarefas. Foi definido um mapa estrutural das informações necessárias à realização da busca pelas Unidades de Saúde.

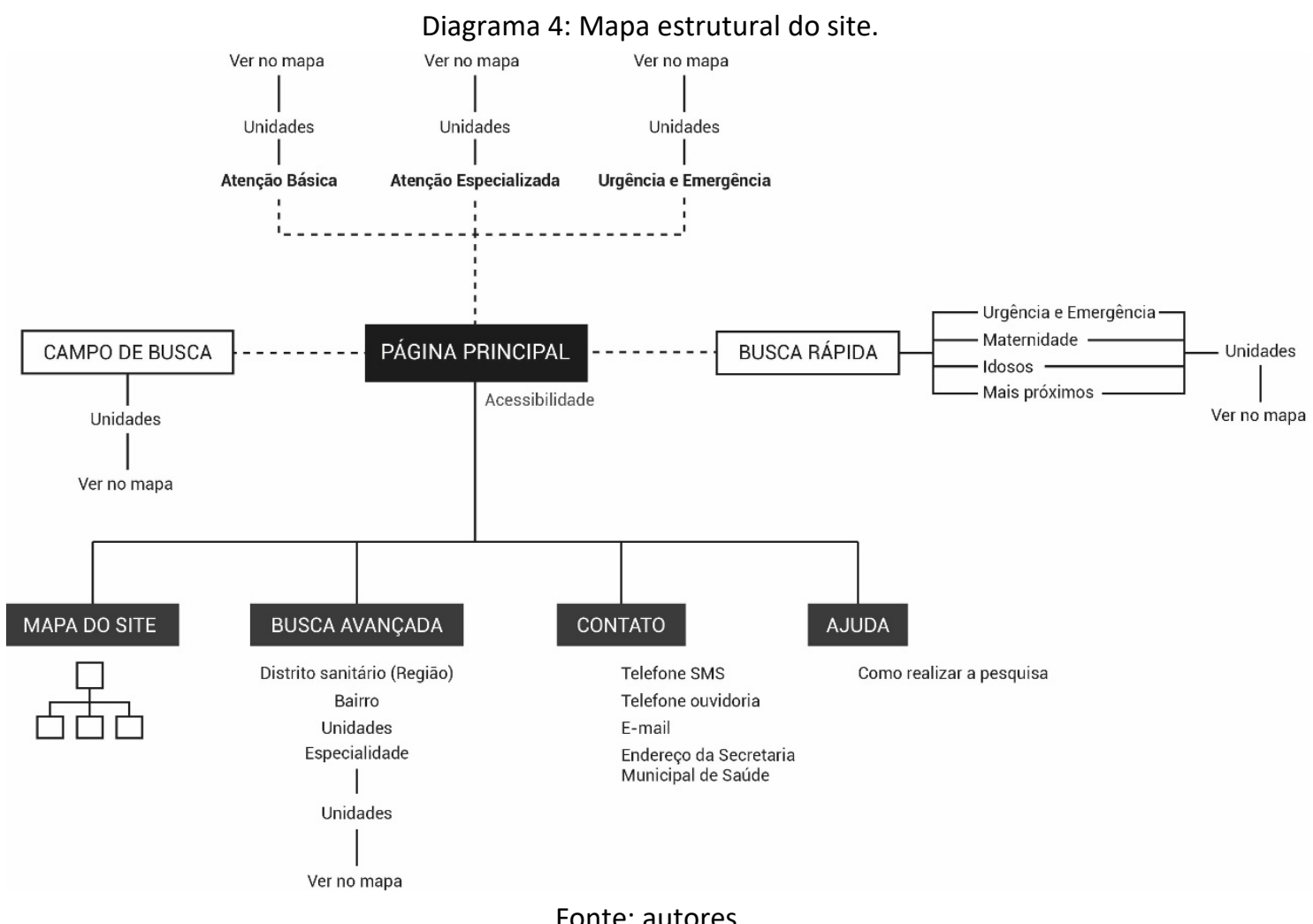


A etapa nomeada Estrutura consistiu da organização estrutural o conteúdo nas telas, por meio da definição dos wireframes, com detalhes específicos do conteúdo, como as informações verbais e não-verbais (Figura 2). A página principal apresenta um cabeçalho formado pelo sinal gráfico e logotipo do site, um menu de navegação e os ícones de acessibilidade no canto superior direito. No corpo do site, o campo de busca está centralizado na página e abaixo, há a visualização dos quatro botões de busca rápida. Posteriormente, há três colunas de texto para explicar a diferença entre os modelos de atendimento do SUS, a Atenção Básica, Atenção Especializada e Urgência e Emergência.

Figura 2: Wireframes das páginas do site proposto.
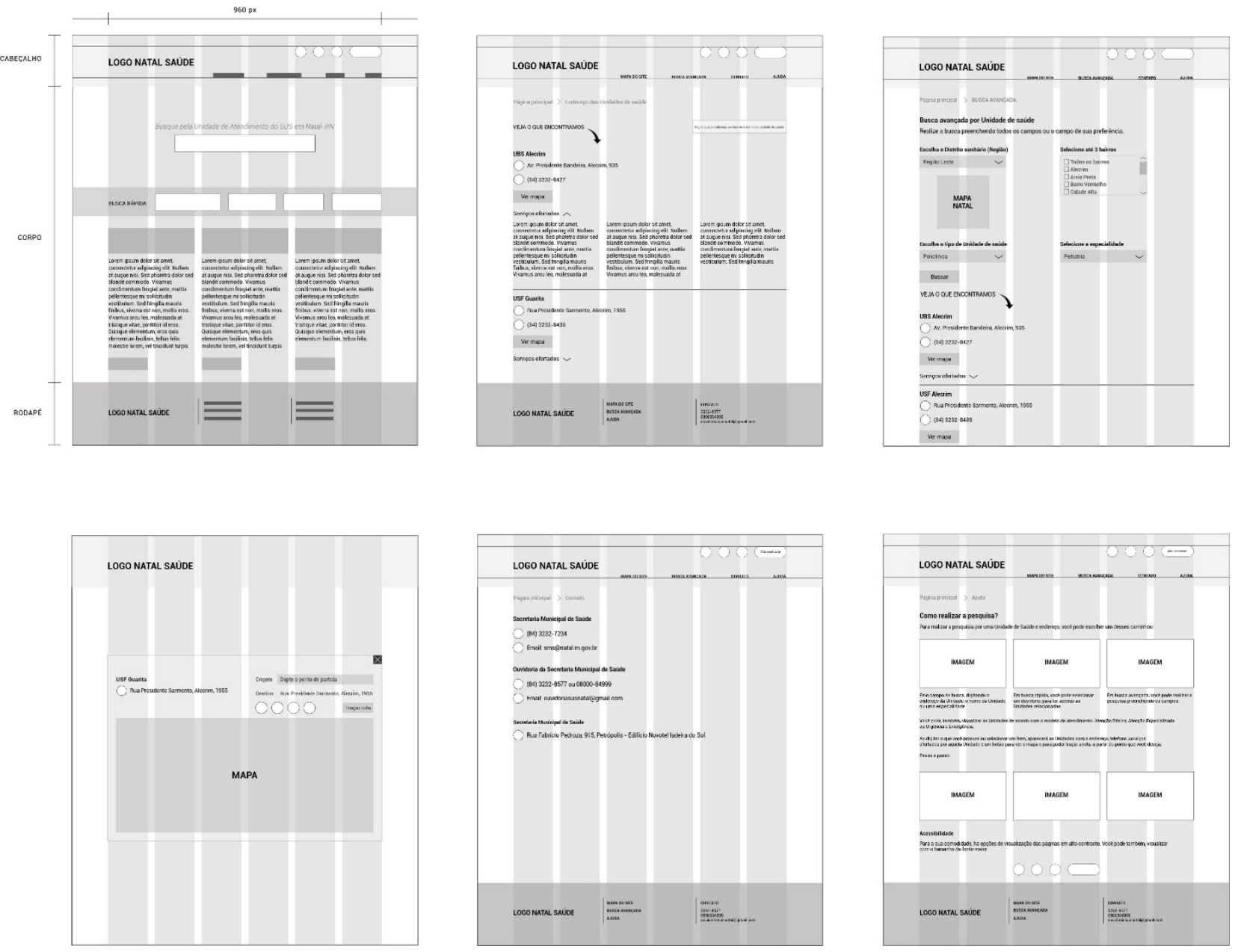

Fonte: autores

Foi estabelecido um diagrama com seis colunas, para possibilitar a diagramação do conteúdo. Os diagramas de múltiplas colunas permitem formatos flexíveis, que integram textos e ilustrações. Pode-se articular a hierarquia, criando zonas específicas para cada tipo de conteúdo (LUPTON, 2006). Textos e imagens podem ocupar uma coluna ou espraiar-se por muitas delas. $\mathrm{Na}$ página principal, foram criadas três áreas de texto distintos, com duas colunas e um botão para ver as Unidades ocupando uma coluna. Estabeleceu-se padrão para organização de informações 
das Unidades, com endereço, telefone e serviços, independente do tipo de busca realizado pelo usuário (no campo de busca, em busca rápida, busca avançada). Assim, os elementos que se repetem estão posicionados no mesmo local, como também, o cabeçalho e o rodapé.

$\mathrm{Na}$ etapa seguinte, denominada Visual, foi definida a composição final do conteúdo, a identidade visual e as interfaces gráficas do website a partir de modelo de baixa fidelidade. É importante ressaltar que o modelo de alta fidelidade (etapa Execução) não foi desenvolvido.

Para que o sistema se adequasse a um conjunto amplo de usuários, foram desenvolvidas interfaces em alto contraste, para se adaptar aos usuários com limitações visuais. Na Internet, a acessibilidade refere-se principalmente às recomendações do e-MAG (Modelo de Acessibilidade em Governo Eletrônico) para o Governo Brasileiro. Visto que o critério de acessibilidade teve como parâmetro a Legislação, foi seguido as recomendações descritas no e-MAG. Além do alto contraste, a interface incluiu funcionalidade que possibilitam a ampliação e redução das letras, o que atendeu os critérios de condução (legibilidade), adaptabilidade, ergonomia e design e acessibilidade.

\section{Considerações finais}

Os diversos artefatos, dispositivos e aplicativos existentes que possibilitam acesso às inúmeras informações, requerem interfaces que privilegiem a interação dos usuários, o que sustenta seus atributos funcionais. Para essa interação ocorrer com eficácia, é necessário que o usuário utilize a interface com eficiência. Assim, a Interação Humano-Computador privilegia o uso desses sistemas interativos, levando em conta as necessidades dos usuários. Nesse sentido, os sistemas interativos devem promover o uso objetivo das interfaces. Para tanto, é possível, a partir de diretrizes que orientem tanto a avaliação como o desenvolvimento de projeto destas interfaces, considerando a experiência do usuário, a usabilidade, o sistema interativo e, finalmente, o design da interface.

A partir do estudo e da análise de autores que abordam tais aspectos relacionados ao design de interfaces, foi possível organizar uma lista de critérios de usabilidade, relevantes para a avaliação de interfaces interativas, como também, para nortear o desenvolvimento de projetos de design. O conjunto de critérios estabelecidos - condução; visibilidade do status do sistema; consistência e padrões; gestão de erros; adaptabilidade; ergonomia e design; redução da sobrecarga de memória de curta duração; carga de trabalho; compatibilidade; acessibilidade estabelece diretrizes essenciais, que se adequam às necessidades específicas dos usuários.

Como mencionado na introdução deste artigo, tais critérios foram utilizados para a avaliação do acesso às informações sobre Unidades de Saúde do Sistema Único de Saúde (SUS) na cidade do Natal disponíveis no portal da Prefeitura deste município. A pesquisa maior, para além da revisão bibliográfica sobre fundamentos relacionados ao design de interação e usabilidade, foi realizada avaliação com usuários do sistema SUS, com intuito de levantar as principais questões e dificuldades relacionadas ao acesso às informações. A partir de então, foi possível estudar o portal e verificar as oportunidades de projeto. O método utilizado para o desenvolvimento seguiu a proposta de Meurer e Szabluk (2010), que tem como base o método de Garrett (2003). O método apresenta seis etapas de projeto, a saber: Estratégia, Escopo, Estrutura, Esqueleto, Visual e Execução. Na primeira etapa do projeto, estratégia, foram estabelecidos critérios de usabilidade, 
de acordo com o apresentado neste artigo, para a avaliação de interfaces. Estes critérios serviram de base para a análise do site da Prefeitura. Outrossim, os critérios nortearam o desenvolvimento de projeto até a etapa de concepção de modelos de baixa fidelidade (Visual). Para a validação do conjunto de critérios, deve-se, a partir deles, fazer avaliação de usabilidade com usuários em modelo de alta fidelidade, como continuidade da pesquisa.

\section{Referências}

BARBOSA, Simone Diniz Junqueira; SILVA, Bruno Santana da. Interação Humano-Computador. Rio de Janeiro: Elsevier, 2010.

BASTIEN, J.M. Christian, SCAPIN; Dominique L. Ergonomic Criteria for the Evaluation of Human Computer Interfaces (version 2.1). Technical Report n. 156, INRIA, mai., 1993.

BENYON, David. Interação humano-computador. 2 ed. São Paulo: Pearson Prentice Hall, 2011. BERTINI, Enrico, GABRIELLI Silvia; KIMANI, Stephen. Appropriating and assessing heuristics for mobile computing. In: Conference on Advanced Visual Interfaces, AVI'06, mai. 2006, Veneza. Proceedings ...... New York: ACM, 2006.

CYBIS, Walter; BETIOL, Adriana Holtz; FAUST, Richard. Ergonomia e Usabilidade: conhecimentos, métodos e aplicações. 2. ed. São Paulo: Novatec Editora, 2010.

GARRETT, Jesse James. The Elements of User Experience: User-centered design for the web. Berkeley, CA: New Riders; AIGA, 2003.

HORN, R. E.. Information design: emergence of a new profession. In: JACOBSON, Robert (ed.). Information design. Cambridge (MA): The MIT Press, 2000.

LUPTON, Ellen. Pensar com tipos: guia para designers, escritores, editores e estudantes. São Paulo: Cosac Naify, 2006.

MACHADO NETO, Olibário José. Usabilidade da interface de dispositivos móveis: heurísticas e diretrizes para o design. 2013. 118 f. Dissertação (Mestrado) - Curso de Curso de Ciências de Computação e Matemática Computacional, Instituto de Ciências Matemáticas e de Computação, Universidade de São Carlos, São Carlos, 2013.

MEURER, Heli; SZABLUK, Daniela. Projeto E: aspectos metodológicos para o desenvolvimento de projetos dígito-virtuais. Ação Ergonômica: Revista Brasileira de Ergonomia, Porto Alegre, v. 5, n. 2, p.1-9, nov. 2010. Disponível em:

<http://www.abergo.org.br/revista/index.php/ae/article/view/85>. Acesso em: 5 jun. 2016.

NIELSEN, Jakob. 10 Usability Heuristics for User Interface Design. 1995. Disponível em: https://www.nngroup.com/articles/ten-usability-heuristics/. Acesso em 23 set. 2016.

NIELSEN, Jakob. Usability engineering. Cambridge, MA: AP Professional, 1993.

PASSOS, Ravi Figueiredo. O design da informação em interfaces de hipermídias. 2008. 86 f. Dissertação (Mestrado) - Curso de Design, Universidade Anhembi Morumbi, São Paulo, 2008.

PORTUGAL, Cristina. Questões complexas do design da informação e de interação. Infodesign:

Revista Brasileira de Design da Informação, São Paulo, v. 7, n. 2, p.1-6, mai. 2010.

PREECE, Jennifer; ROGERS, Yvonne; SHARP, Helen. Design de interação: além da interação humano-computador. 3. ed. Porto Alegre: Bookman, 2013. 
ROYO, Javier. Design digital. São Paulo: Rosari, 2008.

SANTA ROSA, José Guilherme; MORAES, Anamaria de. Avaliação e Projeto no Design de Interfaces. 2. ed. Rio de Janeiro: 2ab, 2012.

SHNEIDERMAN, Ben; PLAISANT, Catherine. Designing the user interface: strategies for effective human-computer-interaction. 4. ed. Boston: Pearson; Addison Wesley Longman, 2005. 